\title{
A Novel Dynamic Method to Improve First-order Natural Frequency for Test Device
}

\author{
Jun Zhang, Yu Tian, Zongjin Ren, Jun Shao, Zhenyuan Jia \\ Faculty of Mechanical engineering, Dalian University of Technology, No.2 Linggong road, 116024, Dalian, China, \\ Zhangj@dlut.edu.cn
}

\begin{abstract}
It is important to improve the natural frequency of test device to improve measurement accuracy. First-order frequency is basic frequency of dynamic model, which generally is the highest vibration energy of natural frequency. Taking vector force test device (VFTD) as example, a novel dynamic design method for improving first-order natural frequency by increasing structure stiffness is proposed. In terms of six degree-of-freedom (DOF) of VFTD, dynamic model of VFTD is built through the Lagrange dynamic equation to obtain theoretical natural frequency and mode shapes. Experimental natural frequency obtained by the hammering method is compared with theoretical results to prove rationality of the Lagrange method. In order to improve the stiffness of VFTD, increase natural frequency and meet the requirement of high frequency test, by using the trial and error method combined with curve fitting (TECF), stiffness interval of meeting natural frequency requirement is obtained. Stiffness of VFTD is improved by adopting multiple supports based on the stiffness interval. Improved experimental natural frequency is obtained with the hammering method to show rationality of the dynamic design method. This method can be used in improvement of first-order natural frequency in test structure.
\end{abstract}

Keywords: Natural frequency, dynamic analysis, Lagrange dynamic method, structure improvement, test device.

\section{INTRODUCTION}

Natural frequency of mechanical system is a core parameter of reflecting dynamic characteristics, which plays an important role in many dynamic research fields such as vibration control, fault diagnosis, and structural optimization design [1]. In a conventional dynamic test, when system natural frequency is closed to external excitation frequency, signal will produce a distortion affecting the dynamic test accuracy. Thus, improving the natural frequency of the test system is a critical factor for improvement of the accuracy of dynamic test.

As a basis of dynamic design, dynamic analysis is a premise of test system to improve natural frequency. The dynamic measured signal is a time-dependent quantity which can be transformed to the frequency domain [2]. With the frequency domain signal, experimental identification of structural dynamics models is usually based on the modal tests [3]. Many articles have made a dynamic analysis of the test system and analyzed the natural frequency. Andreas Albrecht [4] applies a capacitive sensor to detect displacement, studies natural frequency and expands frequency bandwidth by the Kalman filter method. Li Yingjun [5] makes dynamic analysis to six-dimension-force piezoelectric test system for large load-bearing manipulators and obtains the natural frequency of test system by using the hammering method.
Qin Lan [6] designs a parallel six-dimension force/torque sensor using 8 quartz wafers and obtains natural frequency through a method of negative step response. G. Totis [7] proposes a new three-dimensional dynamometer for $\mathrm{CNC}$ lathes. Natural frequency is obtained by the hammering method, and dynamic response curve during cutting $45 \mathrm{C}$ steel is obtained. Jeong-Xueguang Liu [8] designs an inertia electromagnetic actuator for adjusting the natural frequency. Experimental tests of the natural frequency are conducted and studied to overcome the disadvantage of a very narrow bandwidth. A. Scippa [9] compensates dynamic curves based on the Kalman filter to reduce the dynamic error of cutting force test. Junqing Ma [10] proposes a dynamic compensation method to broaden operation frequency based on the frequency response function for two-dimension force sensor. Zongjin Ren [11] proposes a dynamic compensation method based on acceleration for a rocket vector force test system reducing the dynamic error. J. Roj [12] develops real-time dynamic error correction of transducers based on a novel neural network. In terms of all articles above, natural frequency is mainly obtained by experiment and dynamic compensation, but all dynamic methods have to be completed after manufacturing structure, which cannot be used in the design stage. 
Dynamic characteristics of mechanical structure can be modified through structural improvements [13]. In order to make dynamic analysis in the design stage and improve dynamic characteristic through changing structure, a method of theoretical dynamic modeling has been observed. Theoretical dynamic methods commonly include NewtonEuler, Lagrange, Kane equation, energy conservation law, etc. [14]. Mingtao Liu [15] applies the lumped parameter method to build up a novel inner displaced cam to obtain firstorder natural frequency. Sang Won Lee [16] obtains a five degree-of-freedom (DOF) theoretical model of mesoscale machine tools with a centralized parameter method and analyzes its dynamic characteristics. Schmitz [17] predicts dynamic characteristics of high-speed machine tool with the dynamic substructure method. Xiaotian Wei [18] establishes a dynamic model of electric vehicle powerplant mount system with the Lagrange equation. Modal analysis of suspension system is analyzed, respectively, with Adams / Vibration and Matlab, and natural frequency, mode shape is obtained. Xiaohui Jia [19] adopts Lagrange equations to establish modified mechanical dynamic differential equation for 3-PRR flexible parallel mechanism in micro/nanooperation and analysis of natural frequency. Heng Lu [20] establishes dynamic models of nano-micron lathes with centralized parameter method and studies frequency response of machine tool underground vibration. Stiffness is one of the fundamental factors affecting natural frequency. However, theoretical dynamic modeling has seldom analyzed the law of stiffness over natural frequency, and increasing natural frequency by changing stiffness of structure is relatively rare, which is difficult to be applied to dynamic design directly.

As a core component of advanced aircraft, vector force in vector nozzle engine directly controls the posture of aircraft, and accurate measurement of vector force is a critical guarantee for reliable operation of aircraft. To satisfy the requirement of high-frequency measurement, VFTD must satisfy the dynamic characteristics of high-frequency response. Therefore, dynamic design method for improving first-order natural frequency by increasing structure rigidity is proposed. Taking VFTD as example, dynamic analysis and dynamic structure improvement are applied to complete the dynamic design. Firstly, Lagrange is used to build the dynamic model of VFTD based on six DOF. Theoretical natural frequency before improvement and influence of stiffness on first-order natural frequency are obtained. By using the method of trial and TECF, stiffness interval is analyzed satisfying the requirement of natural frequency. Hammering method is designed to obtain experimental frequency to compare with theoretical natural frequency. In order to improve first-order natural frequency, high-stiffness structure is designed and improved natural frequency is obtained. First-order natural frequency improved from $248 \mathrm{~Hz}$ to $463 \mathrm{~Hz}$ meets the high-frequency test requirement of vector force and verifies rationality of the dynamic design method.

\section{Methodology instruction}

A novel method of dynamic design to improve first-order natural frequency is proposed based on stiffness improvement through dynamic design of the model. Considering characteristic in six degree-of-freedom of the model, kinetic modeling of model is completed based on the Lagrange dynamic equation. Equivalent stiffness model is set up, and theoretical natural frequencies of dynamic model are calculated with the modal analysis method. Dynamic calibration method like the hammering method is used to obtain experimental frequency. Theoretical natural frequencies are compared with experimental results to verify rationality of the theoretical dynamic model. Subsequently, influence of structure stiffness on first-order natural frequency is analyzed, and stiffness interval satisfying the required natural frequency is obtained through a method of TECF. Thus, natural frequency can improve by increasing stiffness of structure after analyzing the dynamic spectrum. After improving the structure of dynamic model, dynamic calibration is used to obtain natural frequency of the improved model. By comparing theoretical natural frequency with experimental natural frequency, frequency improvement and rationality of dynamic design method are verified. Flow chart of the method is shown in Fig.1.

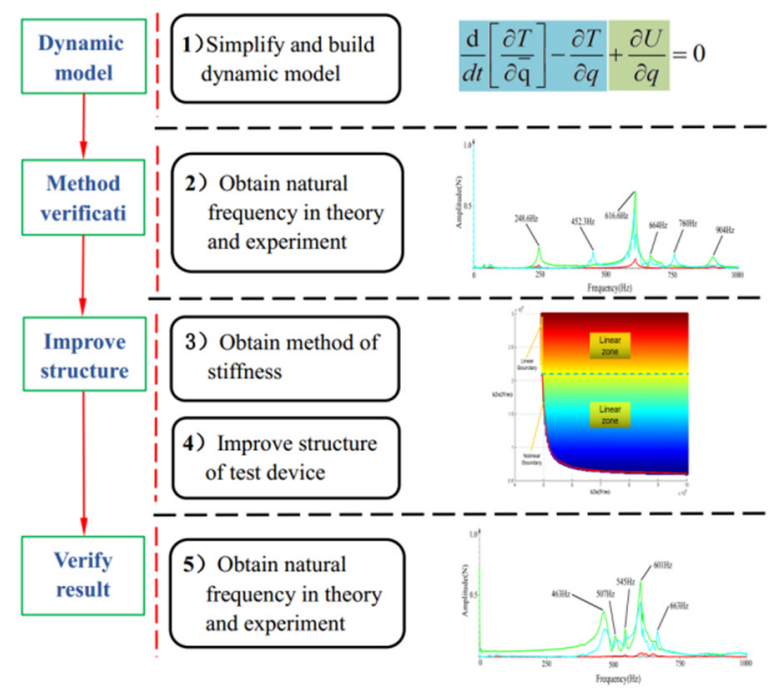

Fig.1. Flow chart of dynamic method.

\section{VECTOR FORCE TEST DEVICE (VFTD)}

Vector force test device is designed to measure vector force shown in Fig.2. When vector force acts on the piezoelectric dynamometer, induced charges generate from the piezoelectric wafer, which can be processed through hardware. Computer is used to obtain output voltages of vector forces. Adopting a piezoelectric wafer as a force sensing element, force vector test device is mainly composed of dynamometer, force vector loading device, lateral loading device and rigid body based on the force measurement requirements of changing angle at high frequency. Force vector loading device and lateral loading device are used only for force static calibration. 


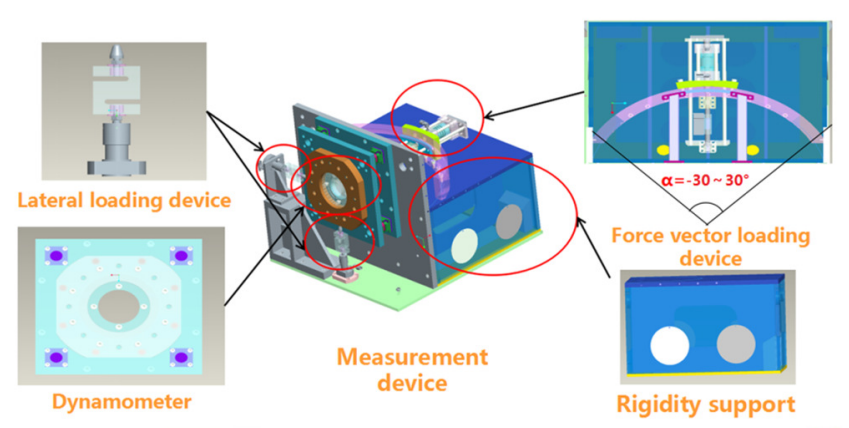

Fig.2. Test device of thrust vector control engine.

Four piezoelectric sensors of dynamometer are arranged in the same positive direction for measuring force in directions of X, Y, and Z. Four sensors' layout is a specific layout with symmetric structure in 2 orthogonal directions, decreasing disturbing error and increasing compensation capability. Piezoelectric sensor has a characteristic of high frequency and stiffness, the stiffness $\mathrm{E}=8 \times 10^{4} \mathrm{~N} / \mathrm{mm}^{2}$, the highest frequency $\mathrm{f}=200 \mathrm{kHz}$, whose upper and lower covering with stainless steel $(1 \mathrm{Cr} 18 \mathrm{NiMoV})$ material is antimagnetic and antirust. The sensors not only simplify the solution process but also compensate for the output deviation due to bending caused by low stiffness and external environmental disturbances. Rigid body is mainly used to ensure positioning accuracy between dynamometer and vector force loading device. The force vector loading device, which can realize vector loading of angle with -30 degrees to 30 degrees, is mainly used to load vector force at different angles. Lateral loading device is used to load lateral force in orthogonal direction.

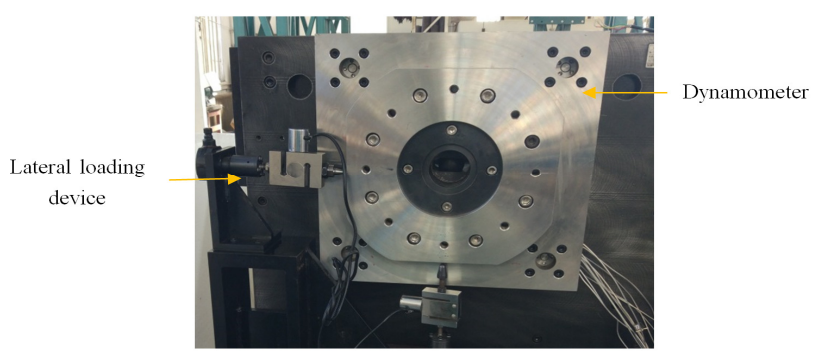

Fig.3. Physical picture of test device.

\section{SIMULATION AND DISCUSSION FOR DYNAMIC METHOD OF VFTD}

\section{A. DYNAMIC EQUATIONS OF VFTD BASED ON THE LAGRANGE METHOD}

Lagrange dynamical equations are used for the kinetic modeling. Lagrange dynamic equation is:

$$
\frac{d}{d t}\left[\frac{\partial T}{\partial \dot{q}_{j}}\right]-\frac{\partial T}{\partial q_{j}}+\frac{\partial U}{\partial q_{j}}=0,
$$

where $T$ is total kinetic energy of system, $U$ is total potential energy of system, $q_{j}$ is generalized coordinates of system, $\dot{q}_{j}$ is generalized velocity of system. According to different loading state and structure, the kinetic model of VFTD is properly simplified based on the model in Fig.2., which is divided into three major parts: rigid support, lower plate of dynamometer and upper plate of dynamometer. The number of three major parts is listed as $1,2,3 . k_{1}$ is bolt stiffness representing connection stiffness between rigid support and the earth, $k_{2}$ is connecting plate stiffness representing connection stiffness between rigid support and lower plate of dynamometer, $k_{3}$ is sensors' stiffness representing connection stiffness between lower plate of dynamometer and upper plate of dynamometer. In order to fully reflect influence of each part in six dimensions on the dynamic characteristics, no DOF in each part can be ignored in the analysis, i.e., each part considers 6-DOF-generalized coordinates. Thus, the system has a total of $18 \mathrm{DOF}$, and generalized coordinates are relative.

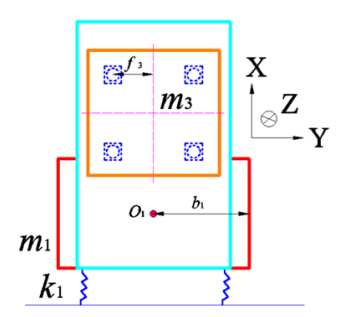

a) main direction

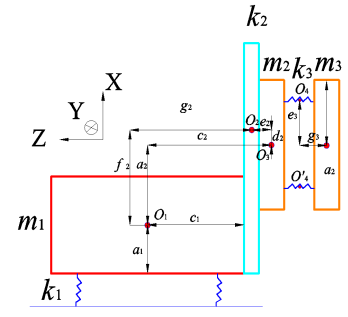

b) lateral direction
Fig.4. Vector force test device dynamics model.

$q_{1 x}$ is displacement of rigid support in $\mathrm{X}$ direction; $q_{1 y}$ is displacement of rigid support in $\mathrm{Y}$ direction; $q_{1 z}$ is displacement of rigid support in $\mathrm{Z}$ direction; $\theta_{1 x}$ is rotation angle of rigid support along $\mathrm{X}$ direction; $\theta_{1 y}$ is rotation angle of rigid support along $\mathrm{Y}$ direction; $\theta_{1 z}$ is rotation angle of rigid support along $\mathrm{Z}$ direction; $q_{2 x}$ is displacement of lower plate of dynamometer in $\mathrm{X}$ direction; $q_{2 y}$ is displacement of lower plate of dynamometer moving in $\mathrm{Y}$ direction; $q_{2 z}$ is displacement of lower plate of dynamometer in $\mathrm{Z}$ direction; $\theta_{2 x}$ is rotation angle of lower plate of dynamometer along $\mathrm{X}$ direction; $\theta_{2 y}$ is rotation angle of lower plate of dynamometer along $\mathrm{X}$ direction; $\theta_{2 z}$ is rotation angle of lower plate of dynamometer along $\mathrm{X}$ direction; $q_{3 x}$ is displacement of upper plate of dynamometer in $\mathrm{X}$ direction; $q_{3 y}$ is displacement of upper plate of dynamometer in $\mathrm{Y}$ direction; $q_{3 z}$ is displacement of upper plate of dynamometer in $\mathrm{Z}$ direction; $\theta_{3 x}$ is rotation angle of upper plate of dynamometer along $\mathrm{X}$ direction; $\theta_{3 y}$ is rotation angle of upper plate of dynamometer along $\mathrm{X}$ direction; $\theta_{3 z}$ is rotation angle of 
upper plate of dynamometer along $\mathrm{X}$ direction. Based on the model in Fig.2., dynamics model of vector force test device is shown in Fig.4.

$\mathrm{O}_{1}, \mathrm{O}_{2}, \mathrm{O}_{3}$, and $\mathrm{O}_{4}$ are, respectively, centroid of rigid support, connecting plate, upper and lower plates of dynamometer and sensor. $a_{1}, b_{1}$, and $c_{1}$ are size parameters in the $\mathrm{X}, \mathrm{Y}$, and $\mathrm{Z}$ directions of rigidity support. $a_{2}, c_{2}$ are centroid distance between rigid support and dynamometer in $\mathrm{X}$ direction, $\mathrm{Z}$ direction. $g_{2}, e_{2}$ are centroid distance between connecting plate and rigid supporter, lower plate of dynamometer in $\mathrm{Z}$ direction. $g_{2}, e_{2}$ are centroid distance between connecting plate and rigid supporting, lower plate of dynamometer in $\mathrm{X}$ direction. $g_{3}$ is centroid distance between sensors and upper plate (lower plate) dynamometer in $\mathrm{Z}$ direction. $e_{3}$ is centroid distance between sensor and upper plate (lower plate) of dynamometer in $\mathrm{X}$ direction. $f_{3}$ is centroid distance between sensor and upper plate dynamometer in $\mathrm{Y}$ direction. $m_{1}, m_{2}$ are quality of dynamometer and rigid support, respectively. $J_{1 \mathrm{i}}, J_{2 \mathrm{i}}(i$ represents $\mathrm{X}, \mathrm{Y}$, and $\mathrm{Z}$ direction) are moment of inertia of dynamometer and rigid support, respectively. Based on (1), it is important for solving dynamic equations to determine the total kinetic energy and total potential of the system. Kinetic energy is considered of 6-DOF in dynamometer, rigidity support. Based on dynamic model and its dynamic equation, kinetic energy of rigid support $T_{1}$, kinetic energy of upper plate of dynamometer $T_{2}$, kinetic energy of lower plate of dynamometer $T_{3}$ and its total kinetic energy $T$ are respectively:

$$
\begin{aligned}
& T_{1}=\frac{1}{2}\left(J_{1 x}{\omega_{1 x}}^{2}+J_{1 y}{\omega_{1 y}}^{2}+J_{1 z}{\omega_{1 z}}^{2}+m_{1} v_{1 x}{ }^{2}+m_{1} v_{1 y}{ }^{2}+m_{1} v_{1 z}{ }^{2}\right) \\
& T_{2}=\frac{1}{2}\left[J_{2 x} \omega_{2 x}{ }^{2}+J_{2 y} \omega_{2 y}{ }^{2}+J_{2 z} \omega_{2 z}{ }^{2}+m_{2}\left(v_{2 z}-a_{2} \omega_{1 y}\right)^{2}+\right. \\
& \left.m_{2}\left(v_{2 x}-c_{2} \omega_{1 y}\right)^{2}+m_{2}\left(v_{2 y}+c_{2} \omega_{1 x}+a_{2} \omega_{1 z}\right)^{2}\right] \\
& T_{3}=\frac{1}{2}\left[J_{3 x} \omega_{3 x}{ }^{2}+J_{3 y} \omega_{3 y}{ }^{2}+J_{3 z} \omega_{3 z}{ }^{2}+m_{3}\left(v_{3 x}-c_{3} \omega_{2 y}\right)^{2}+\right. \\
& \left.m_{3}\left(v_{3 y}+c_{3} \omega_{2 x}\right)^{2}+m_{3} v_{3 z}{ }^{2}\right] \\
& T=T_{1}+T_{2}+T_{3}
\end{aligned}
$$

where $\omega_{i j}$ and $v_{i j}$ are angular acceleration and velocity of the $i$ part in $j$ direction. As for potential energy $U$, this paper mainly analyzes elastic potential energy of rigid support and the earth $U_{1}$, elastic potential energy of dynamometer and rigid support $U_{2}$, elastic potential energy of dynamometer $U_{3}$ and the total elastic potential energy $U$ are respectively:

$$
\begin{aligned}
& U_{1}=\frac{1}{2}\left[k_{1 x}\left(q_{1 x}+c_{1} \theta_{1 y}+b_{1} \theta_{1 z}\right)^{2}+k_{1 x}\left(q_{1 x}-c_{1} \theta_{1 y}+b_{1} \theta_{1 z}\right)^{2}+\right. \\
& k_{1 x}\left(q_{1 x}+c_{1} \theta_{1 y}-b_{1} \theta_{1 z}\right)^{2}+k_{1 x}\left(q_{1 x}-c_{1} \theta_{1 y}-b_{1} \theta_{1 z}\right)^{2}+k_{1 y}\left(q_{1 y}\right. \\
& \left.+a_{1} \theta_{1 z}+c_{1} \theta_{1 x}\right)^{2}+k_{1 y}\left(q_{1 y}-a_{1} \theta_{1 z}+c_{1} \theta_{1 x}\right)^{2}+k_{1 y}\left(q_{1 y}+a_{1} \theta_{1 z}\right. \\
& \left.-c_{1} \theta_{1 x}\right)^{2}+k_{1 y}\left(q_{1 y}-a_{1} \theta_{1 z}-c_{1} \theta_{1 x}\right)^{2}+k_{1 z}\left(q_{1 z}+a_{1} \theta_{1 y}+b_{1} \theta_{1 x}\right)^{2} \\
& +k_{1 z}\left(q_{1 z}-a_{1} \theta_{1 y}+b_{1} \theta_{1 x}\right)^{2}+k_{1 z}\left(q_{1 z}-a_{1} \theta_{1 y}-b_{1} \theta_{1 x}\right)^{2}+k_{1 z}\left(q_{1 z}\right. \\
& \left.\left.+a_{1} \omega_{1 y}-b_{1} \omega_{1 x}\right)^{2}\right]
\end{aligned}
$$

$$
\begin{aligned}
& U_{2}=\frac{1}{2}\left[k_{2 x}\left(-q_{1 x}+q_{2 x}+e_{2} \theta_{2 y}+g_{2} \theta_{1 y}\right)^{2}+k_{2 y}\left(-q_{1 y}+q_{2 y}+\right.\right. \\
& \left.\left.d_{2} \theta_{2 z}-f_{2} \theta_{1 z}-e_{2} \theta_{2 x}-g_{2} \theta_{1 x}\right)^{2}+k_{2 z}\left(-q_{1 z}+q_{2 z}-d_{2} \theta_{2 y}+f_{2} \theta_{1 y}\right)^{2}\right] \\
& U_{3}=\frac{1}{2}\left\{k _ { 3 x } \left[\left(-q_{2 x}+q_{3 x}+g_{3}\left(\theta_{2 y}+\theta_{3 y}\right)+f_{3}\left(-\theta_{2 z}+\theta_{3 z}\right)\right)^{2}+\right.\right. \\
& \left(-q_{2 x}+q_{3 x}-g_{3}\left(\theta_{2 y}+\theta_{3 y}\right)+f_{3}\left(-\theta_{2 z}+\theta_{3 z}\right)\right)^{2}+\left(-q_{2 x}+q_{3 x}\right. \\
& \left.+g_{3}\left(\theta_{2 y}+\theta_{3 y}\right)-f_{3}\left(-\theta_{2 z}+\theta_{3 z}\right)\right)^{2}+\left(-q_{2 x}+q_{3 x}-g_{3}\left(\theta_{2 y}+\right.\right. \\
& \left.\left.\left.\theta_{3 y}\right)-f_{3}\left(-\theta_{2 z}+\theta_{3 z}\right)\right)^{2}\right]+k_{3 y}\left[\left(-q_{2 y}+q_{3 y}+g_{3}\left(-\theta_{2 x}-\theta_{3 x}\right)\right.\right. \\
& \left.+e_{3}\left(-\theta_{2 z}+\theta_{3 z}\right)\right)^{2}+\left(-q_{2 y}+\left(-q_{2 y}+q_{3 y}-g_{3}\left(-\theta_{2 x}-\theta_{3 x}\right)-\right.\right. \\
& \left.\left.e_{3}\left(-\theta_{2 z}+\theta_{3 z}\right)\right)^{2}\right]+k_{3 z}\left[\left(-q_{2 z}+q_{3 z}+f_{3}\left(\theta_{2 x}-\theta_{3 x}\right)+e_{3}\left(\theta_{2 y}\right.\right.\right. \\
& \left.\left.-\theta_{3 y}\right)\right)^{2}+\left(-q_{2 z}+q_{3 z}-f_{3}\left(\theta_{2 x}-\theta_{3 x}\right)+e_{3}\left(\theta_{2 y}-\theta_{3 y}\right)\right)^{2}+ \\
& \left(-q_{2 z}+q_{3 z}+f_{3}\left(\theta_{2 x}-\theta_{3 x}\right)-e_{3}\left(\theta_{2 y}-\theta_{3 y}\right)\right)^{2}+\left(-q_{2 z}+q_{3 z}\right. \\
& \left.\left.\left.-f_{3}\left(\theta_{2 x}-\theta_{3 x}\right)-e_{3}\left(\theta_{2 y}-\theta_{3 y}\right)\right)^{2}\right]\right\} \\
& U=U_{1}+U_{2}+U_{3},
\end{aligned}
$$

where $k_{1 i} k_{2 i} k_{3 i}$ are, respectively, rigidity of rigidity support and the earth, dynamometer and rigid support, upper and lower plates of dynamometer in different directions. Total kinetic energy $T$ and potential energy $U$ are substituted into (1). The equation after simplification is obtained as:

$$
M \ddot{q}+K q=0,
$$

$M$ is a mass matrix simplified by kinetic energy $T . K$ is stiffness matrix simplified by elastic potential energy $U . \ddot{q}$ $q$ are, respectively, acceleration vector and displacement vector, whose dimension is $18 \times 1$. Supposing $q=\phi \sin (\omega t+\theta)$, it is substituted into (7). After simplification, the following equation is obtained:

$$
K \phi_{i}=\omega_{i}^{2} M \phi_{i}
$$

$\omega_{i}$ is ith-order angular frequency. $\phi_{i}$ is ith-order mode vector. Therefore, natural frequencies and modes of each order can be obtained by (8).

\section{B. STIFFNESS MODEL OF VFTD}

In order to improve the result of system dynamic analysis, it is very important to determine the stiffness of bolts $\left(k_{1}\right)$, connecting plate $\left(k_{2}\right)$, and sensors $\left(k_{3}\right)$. Based on the method of theoretical stiffness modeling, the stiffness in main direction ( $Z$ direction) $k_{z}$ of each part can be obtained by using tension and compression stiffness model as shown in (9). Lateral stiffness (X,Y direction) $k_{x}, k_{y}$ are obtained by using shear model as shown in (10). 


$$
\begin{gathered}
k_{z}=\frac{F}{\Delta_{z}}=\frac{\sigma S}{l \varepsilon}=\frac{E S}{l}, \\
k_{x}=\frac{F}{\Delta_{x}}=\frac{\tau S}{l \gamma}=\frac{G S}{l}=\frac{E S}{2 l(1+\mu)},
\end{gathered}
$$

\section{1) Sensor stiffness $k_{3}$}

Sensor is mainly made up of lower block, upper block and force sensitive components. The material of upper and lower plate is Q235, and the material of sensor is $\mathrm{SiO}_{2}$. Supposing $k_{31}, k_{32}, k_{33}$ are, respectively, upper plate of sensor, force sensing element and lower plate of sensor, which are connected in series on the structure. Fig.5. is series-parallel connection of $k_{3}$ and (11) is sensors' equivalent stiffness.

$$
\begin{aligned}
& k_{3}=\frac{1}{\frac{1}{k_{31}}+\frac{1}{k_{32}}+\frac{1}{k_{33}}} \text {, } \\
& \begin{array}{l}
F_{1} \\
\left.k_{31}\right\} \\
\left.k_{32}\right\} \\
\left.k_{33}\right\}
\end{array}
\end{aligned}
$$

Fig.5. Sensor model of stiffness connection.

Upper and lower sensor plates and force sensitive elements are subjected to tension and shear force through mechanical analysis. The models in the main and the lateral direction are tension and shear model calculated according to (9), (10), respectively.

2) Stiffness of connecting plate $k_{2}$

Connecting plate can be regarded as cantilever beam, and its main stiffness is calculated as:

$$
k_{2 z}=\frac{F}{\omega}=\frac{3 E I}{a^{3}},
$$

where $\omega$ is deflection of force loading point. a is arm of force from force loading point, $I$ is moment of inertia of beam. The lateral stiffness is still calculated as shear model by $(10)$.

3) Stiffness of rigid support connecting ground $k_{3}$

Rigid support and the earth mainly use bolts to complete connection, considering connection stiffness between bolts and the earth. Connection stiffness of the earth mainly considers stiffness of bolts. Bolt connections have both elastic and damping characteristics. This characteristic will reduce overall structural stiffness and increase damping, resulting in decrease of natural frequency of machine tool. Connection stiffness has a significant effect on overall dynamic performance [21]. Considering influence of torque caused by deflection and overturning of the test device, bolts are arranged in 4-point square layout. Equations (9) and (10) are applied in tension and shear models, respectively.

\section{RESULTS AND ANALYSIS OF NATURAL FREQUENCY}

Based on stiffness matrix, mass matrix and (8), natural frequencies and mode shapes solved are as given in Table 1.

Table 1. Theoretical natural frequencies and mode shapes.

\begin{tabular}{|c|c|c|}
\hline Order number & $\begin{array}{c}\text { Theoretical } \\
\text { frequency [Hz] }\end{array}$ & Mode shape \\
\hline 1 & 253 & $\begin{array}{c}\text { Vibration of } \\
\text { dynamometer in } Z \\
\text { direction }\end{array}$ \\
\hline 2 & 675 & $\begin{array}{c}\text { Vibration of } \\
\text { dynamometer in } X \\
\text { direction }\end{array}$ \\
\hline 3 & 765 & $\begin{array}{c}\text { Rotation of } \\
\text { dynamometer and rigid } \\
\text { support along } Z \text { axis }\end{array}$ \\
\hline 4 & 895 & $\begin{array}{c}\text { Rotation of rigid } \\
\text { support along Y axis }\end{array}$ \\
\hline 5 & Vupportion of rigid $Z$ direction \\
\hline
\end{tabular}

In order to verify theoretical modal analysis results, the hammering test method is adopted in dynamic calibration shown in Fig.6. Dynamic test system mainly consists of dynamometer, hammer, charge amplifier (Kistler 5018), data acquisition (data translation DT9804), computer and software.

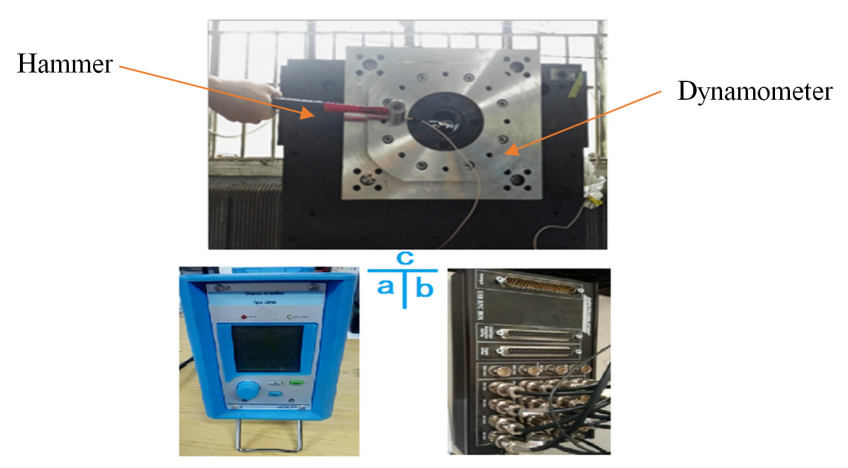

Fig.6. Dynamic test device.

a) charge amplifier b) data acquisition c) test device

Hammering test method is a dynamic calibration adopting hammer as a stimulating device. When force sensor is hammered at a certain speed, charges generated by force sensor are transmitted to computer through the signal acquisition system. Software is used for FFT and filtering analysis of dynamic signal, and natural frequency curve is obtained as shown in Fig.7. 
Table 2. Comparison of theoretical and experimental natural frequencies.

\begin{tabular}{|c|c|c|}
\hline $\begin{array}{c}\text { Order } \\
\text { number }\end{array}$ & $\begin{array}{c}\text { Theoretical natural } \\
\text { frequency [Hz] }\end{array}$ & $\begin{array}{c}\text { Experimental natural } \\
\text { frequency [Hz] }\end{array}$ \\
\hline 1 & 253 & 248.6 \\
\hline 2 & 475 & 452.3 \\
\hline 3 & & 616.6 \\
\hline 4 & 683 & 664 \\
\hline 5 & 765 & 760 \\
\hline 6 & 895 & 904 \\
\hline
\end{tabular}

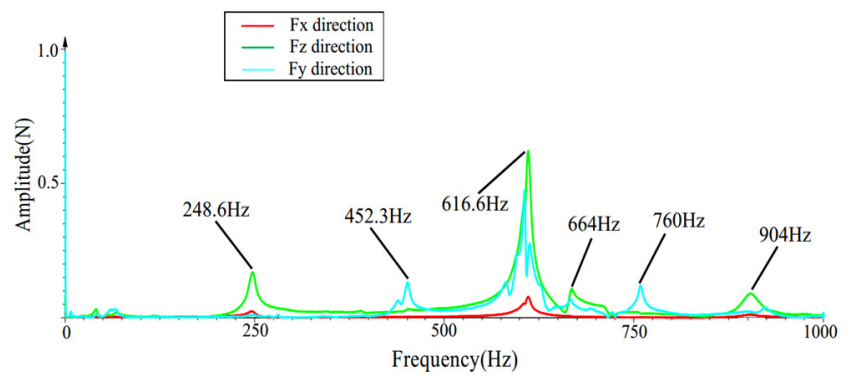

Fig.7. Natural frequency curve.

Analyzing theoretical and experimental natural frequencies, natural frequencies of the two methods are very close. For the most critical first-order natural frequency, the difference between theoretical and experimental method is only $4.4 \mathrm{~Hz}$, accounting for $1.77 \%$ of experiment frequency. For otherorder natural frequencies, theoretical and experimental difference of the second, fourth, fifth, and sixth order are 22.7, 19,5 , and $19 \mathrm{~Hz}$, respectively, accounting for $5.02 \%, 2.86 \%$, $0.07 \%$, and $2.1 \%$ of respective experiment frequency. In addition, third-order experiment results in a natural frequency of $616.6 \mathrm{~Hz}$ cannot match with theoretical natural frequency because theoretical model must be simplified to save calculation duration and cannot simulate theoretical experimental environment, consider mechanical errors and other factors. However, compared with experimental results, errors between theoretical and experimental models are small, which proves the rationality of theoretical model.

\section{FIRST-ORDER NATURAL FREQUENCY IMPROVEMENT OF VFTD}

According to experimental analysis, first-order natural frequency of VFTD is $248.6 \mathrm{~Hz}$. Taking US F-22 Raptor fighter as example, if the nozzle deflection speed is $20 \%$, and nozzle deflection speed accuracy is $0.1^{\circ}$, excitation frequency is $200 \mathrm{~Hz}$ and first-order natural frequency must be higher than $200 \mathrm{~Hz}$. First-order natural frequency of VFTD is only $48.6 \mathrm{~Hz}$ higher than excitation frequency, which is so small that VFTD is prone to resonance. Thus, stiffness of VFTD needs to be improved. In order to meet the test requirements, actual first-order natural frequency should be at least more than twice the test frequency. This paper set $400 \mathrm{~Hz}$ as required first-order natural frequency.

According to Table 1., because mode shape of first-order natural frequency is vibration of dynamometer in $\mathrm{Z}$ direction, focus should be given to improvement of stiffness in $Z$ direction associated with dynamometer, namely, stiffness of sensors in $\mathrm{Z}$ direction $\left(k_{3 z}\right)$ and that of connecting plate in $\mathrm{Z}$ direction $\left(k_{2 z}\right)$. Improvement of stiffness of sensors needs to change the structure of sensor, which affects accuracy of test and increases difficulty of calculating the problem. Moreover, through theoretical calculation of previous section, connection stiffness between dynamometer and plate is only $1.56 \times 10^{8} \mathrm{~N} / \mathrm{m}$, which is relatively small. Therefore, in order to improve natural frequency, this section is to improve stiffness through improvement of $k_{2 z}$. Effect of $k_{2 z}$ on first-order natural frequency of VFTD is analyzed and shown in Fig.8.

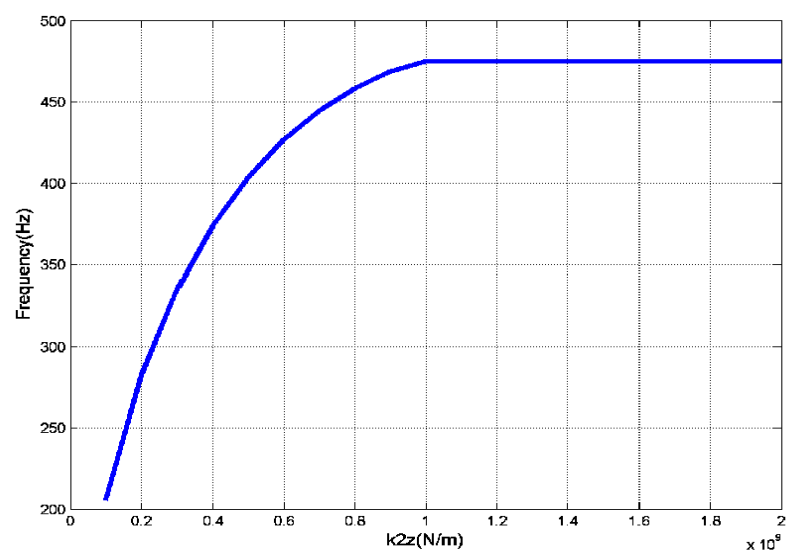

Fig.8. First-order natural frequency of vector force test device over stiffness: abscissa axis is interval of $k_{2 z}$ from $0-2 \times 10^{9} \mathrm{~N} / \mathrm{m}$.

First-order natural frequency increases with $k_{2 z}$ and finally converges to $464 \mathrm{~Hz}$. When stiffness is bigger than $109 \mathrm{~N} / \mathrm{m}$, first-order natural frequency approaches the maximum of $464 \mathrm{~Hz}$. When stiffness is bigger than $0.5 \times 10^{9} \mathrm{~N} / \mathrm{m}$, first-order natural frequency is bigger than the required first-order natural frequency of $400 \mathrm{~Hz}$. Therefore, in terms of maintaining other stiffness, stiffness of $k_{2 z}$ in design should be bigger than $0.5 \times 10^{9} \mathrm{~N} / \mathrm{m}$ to make natural first-order frequency bigger than $400 \mathrm{~Hz}$. However, it is impossible to maintain stiffness of other two directions while changing one-direction stiffness in mechanical design. Therefore, stiffness interval of $k_{2 x}, k_{2 y}, k_{2 z}$ should be simultaneously analyzed to meet the requirements of firstorder natural frequency. Assuming that $f\left(k_{2 x}, k_{2 y}, k_{2 z}\right)$ is first-order natural frequency changing with $k_{2 x}, k_{2 y}, k_{2 z}$, and $f\left(k_{2 x}, k_{2 y}, k_{2 z}\right)$ should be:

$$
f\left(k_{2 x}, k_{2 y}, k_{2 z}\right)>400,
$$


Because mapping of first-order natural frequency is very complicated, it is hard to obtain the interval of independent variable through the direct solution method. Thus, the method of TECF should be used to solve this problem. The detailed steps are as follows:

1) Initial interval of independent variables is determined according to practical requirement.

2) Each variable is taken into (13), and (13) is identified as true or false. If (13) is true, corresponding variable is recorded. If not, variable is not recorded.

3) Curve (or surface) of the recorded variable is drawn.

4) Interval of independent variables is determined with the curve fitting method.

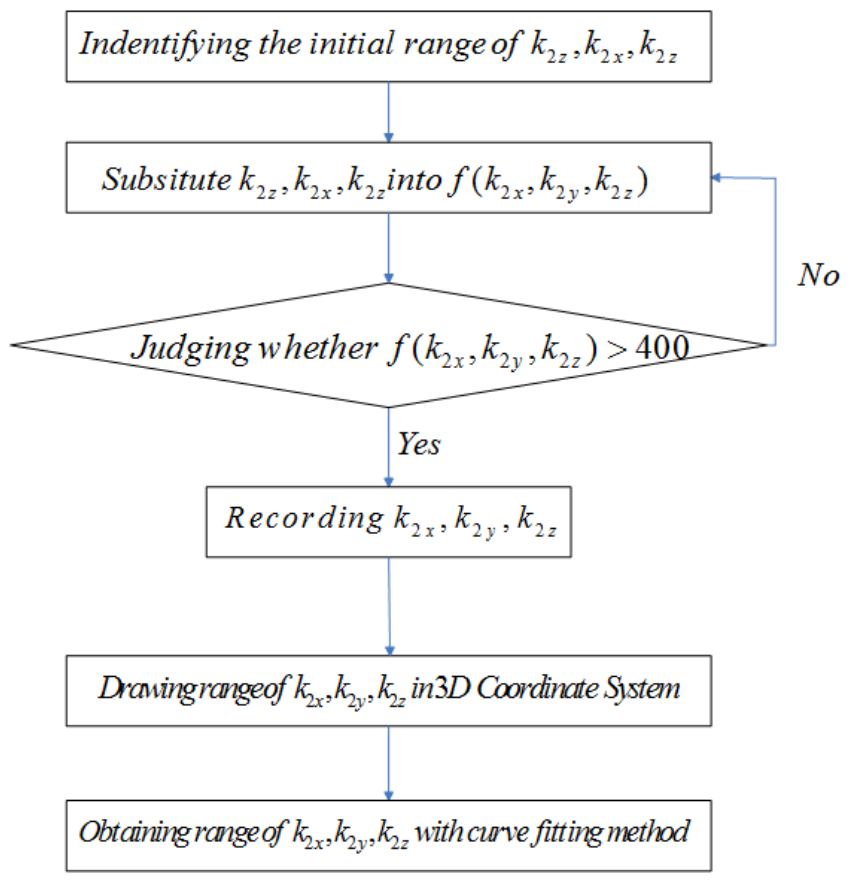

Fig.9. Steps for stiffness interval of $k_{2 x}, k_{2 y}, k_{2 z}$.

According to the method of TECF, it is determined that initial interval of $k_{2 x}, k_{2 y}, k_{2 z}$ is $0-10^{9} \mathrm{~N} / \mathrm{m}$, and interval curve of $k_{2 x}, k_{2 y}, k_{2 z}$ with first-order natural frequency which is bigger than $400 \mathrm{~Hz}$ is shown as follows:

As shown in Fig.10.a), the section $k_{2 x}, k_{2 y}, k_{2 z}$ along $k_{2 y}$ stiffness direction is basically similar to a closed half parabola. Based on shape characteristics of the main body, the law of interval can be explored from the perspective of plane of three stiffness, i.e., intervals in plane of $k_{2 x}, k_{2 y}, k_{2 y}, k_{2 z}$, and $k_{2 z}, k_{2 x}$ are shown in Fig.10.b), Fig.10.c), Fig.10.d), respectively. From stiffness interval of $k_{2 y}, k_{2 z}$, first-order natural frequency meets the requirements of $400 \mathrm{~Hz}$ when $k_{2 y}$ is bigger than $7.75 \times 10^{8} \mathrm{~N} / \mathrm{m}$. As for interval of $k_{2 z}, k_{2 x}$, stiffness interval boundary of $k_{2 z}, k_{2 x}$ is not a simple curve, so the curve should be fitted to investigate the law of interval. It is found that the boundary curve consists of two segments, including linear boundary and non-linear boundary, and thus the interval can be divided into linear zone and nonlinear zone. Boundary of linear domain is obviously a linear boundary, that is, when $k_{2 x}>2.106 \times 10^{9} \mathrm{~N} / \mathrm{m}, k_{2 x}>4.95 \times 10^{8} \mathrm{~N} / \mathrm{m}$ satisfy first-order natural frequency requirement. Boundary of non-linear domain is similar to normal curve. Thus, Gaussian fitting is used to fit in non-linear domain. Gaussian fitting curve is:

$$
G(x)=\sum_{i=1}^{n} A_{i} e^{-\left(\left(x-B_{i}\right)^{2} / C_{i}\right)^{2}},
$$

where $G(x)$ is Gaussian function. $A_{i}, B_{i}, C_{i}$ are fitting coefficients of $i$ th Gaussian curve, respectively. ( $k_{2 z}$, $k_{2 x}$ ) of 1000 points are used for fitting. Three Gaussian fitting functions are chosen through analysis with experimental data. After fitting, correlations that $\mathrm{R}^{2}=0.9994$ and $\mathrm{RMSE}=0.054 \times 108$ are both small, showing high fitting accuracy.

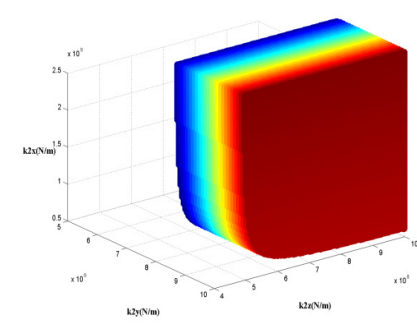

a) Interval of $k_{2 x}, k_{2 y}, k_{2 z}$
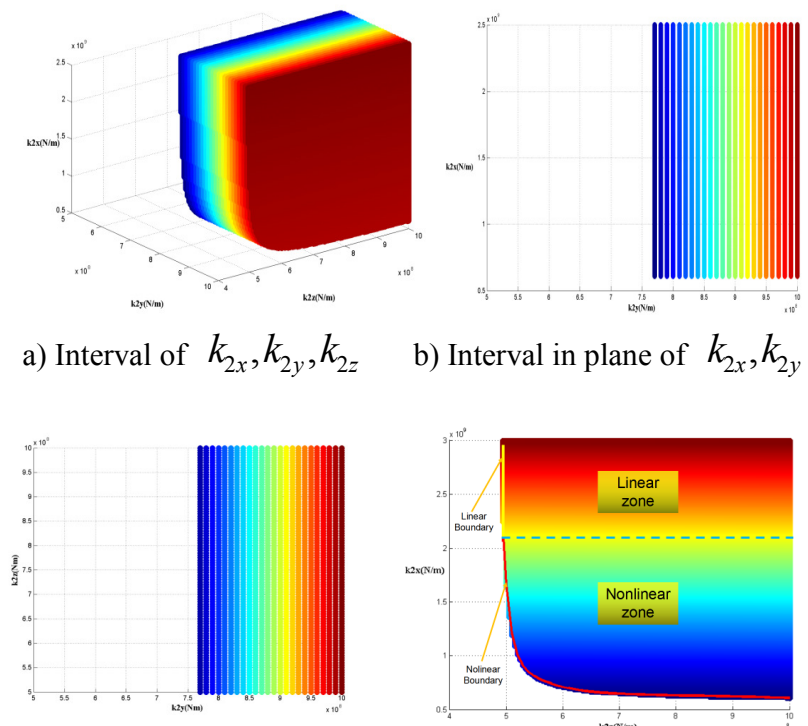

b) Interval in plane of $k_{2 x}, k_{2 y}$

c) Interval in plane of $k_{2 y}, k_{2 z}$ d) Interval in plane of $k_{2 z}, k_{2 x}$

Fig.10. Stiffness interval of $k_{2 x}, k_{2 y}, k_{2 z}$ with first-order natural frequency bigger than $400 \mathrm{~Hz}$.

Fitting coefficients are $A_{1}=5.976 \times 1022, \quad B_{1}=-1.543 \times 108$, $C_{1}=1.154 \times 108, A_{1}=3.131 \times 1013, B_{1}=-1.042 \times 108, C_{1}$ $=4.595 \times 108, \quad A_{1}=6.32 \times 108, \quad B_{1}=7.513 \times 108, \quad C_{1}$ $=1.291 \times 109$, respectively. $\quad k_{2 x}<2.106 \times 10^{9} \mathrm{~N} / \mathrm{m}$ and $k_{2 x}>G\left(k_{2 z}\right)$ satisfy the requirement that first-order natural frequency is bigger than $400 \mathrm{~Hz}$. Stiffness intervals analysis with first-order natural frequency which is bigger than $400 \mathrm{~Hz}$ is summarized, and $k_{2 x}, k_{2 y}, k_{2 z}$ should satisfy following equations: 


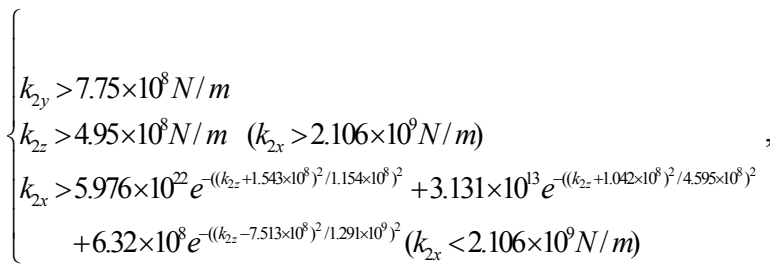

Based on the above analysis, this paper aims to improve first-order natural frequency for improving $k_{2}$. By designing inclined support, side support, and support column shown in Fig.11., stiffness of connecting plate $k_{2}$ can be improved. Series-parallel model is shown below using the stiffness method given in chapter 3 .

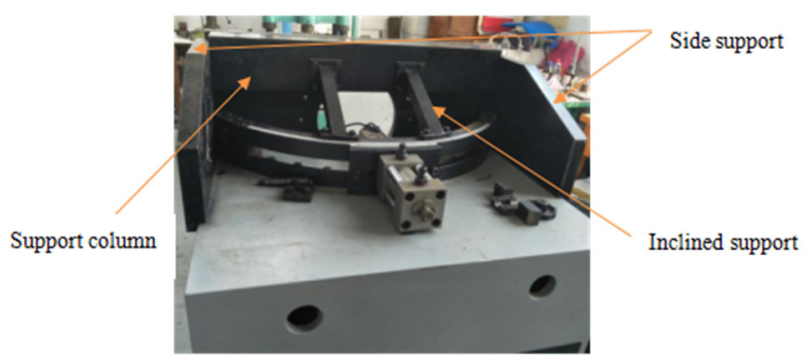

Fig.11. Improved device.

Stiffness of support, inclined support, side plate support and connecting plate are set as $k_{a}, k_{b}, k_{c}, k_{d}$, respectively. Connecting plate is connected with support column, inclined support and side support. Therefore, series-parallel model of $k_{2}$ has changed from cantilever beam model to tension, and compression model in $\mathrm{Z}$ direction to increase $k_{2 z}$ greatly is shown in Fig.12. Improved equivalent stiffness $k_{2}$ is given by (16).

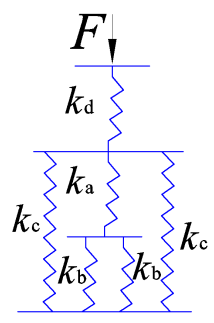

Fig.12. Stiffness connection model of improved structure.

$$
k_{2}=\frac{1}{\frac{1}{k_{d}}+\frac{1}{\frac{1}{2 k_{c}}+\frac{1}{\frac{1}{k_{a}}+\frac{1}{2 k_{b}}}}}
$$

Equations (9) and (10) are applied in tension ( $\mathrm{Z}$ direction) and shear models (X/Y direction), respectively. After calculation, $k_{2 z} k_{2 x} k_{2 y}$ are, respectively, $3.71 \times 10^{9} \mathrm{~N} / \mathrm{m}$,
$9.4 \times 10^{9} \mathrm{~N} / \mathrm{m}, 3.38 \times 10^{9} \mathrm{~N} / \mathrm{m}$, satisfying (15). Based on (8) and dynamic experiments, improved theoretical natural frequencies and mode shapes are shown in Table 3., and improved theoretical and experimental natural frequencies are shown in Table 4. Improved natural frequency curve is obtained as shown in Fig.13.

Table 3. Improved theoretical natural frequencies and mode shapes.

\begin{tabular}{|c|c|c|}
\hline $\begin{array}{c}\text { Order } \\
\text { number }\end{array}$ & $\begin{array}{c}\text { Theoretical } \\
\text { frequency [Hz] }\end{array}$ & Vibration type \\
\hline 1 & 457 & $\begin{array}{c}\text { Rotation of dynamometer } \\
\text { along } Z \text { axis }\end{array}$ \\
\hline 2 & 509 & $\begin{array}{c}\text { Rotation of dynamometer } \\
\text { and rigid support in Z axis }\end{array}$ \\
\hline 3 & 645 & $\begin{array}{c}\text { Vibration of dynamometer } \\
\text { in Z direction }\end{array}$ \\
\hline 4 & 681 & $\begin{array}{c}\text { Rotation of dynamometer } \\
\text { and rigid support along Z } \\
\text { axis and vibration of } \\
\text { dynamometer in Y direction }\end{array}$ \\
\hline
\end{tabular}

Table 4. Improved theoretical and experimental natural frequencies.

\begin{tabular}{|c|c|c|}
\hline $\begin{array}{c}\text { Order } \\
\text { number }\end{array}$ & $\begin{array}{c}\text { Theoretical frequency } \\
{[\mathbf{H z}]}\end{array}$ & $\begin{array}{c}\text { Natural frequency of } \\
\text { test ([Hz]) }\end{array}$ \\
\hline 1 & 457 & 463 \\
\hline 2 & 509 & 507 \\
\hline 3 & & 545 \\
\hline 4 & 645 & 601 \\
\hline 5 & 681 & 663 \\
\hline
\end{tabular}

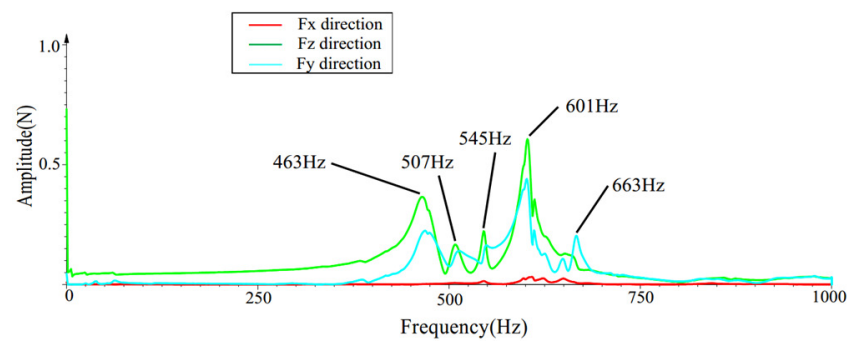

Fig.13. Improved natural frequency curve.

As shown in Table 3. and Table 4., analyzing theoretical and experimental natural frequencies, natural frequencies of the two methods are still close after improvement. Among the most critical first-order natural frequency, difference between theoretical and experimental natural frequency is only $6 \mathrm{~Hz}$, accounting for $1.3 \%$ of experimental frequency. For other natural frequencies, theoretical and experimental difference of the second, fourth, and fifth order are, respectively, 2, 44, and $18 \mathrm{~Hz}$, accounting for $0.4 \%, 7.8 \%$ and $2.7 \%$ of 
corresponding experimental frequency. In addition, thirdorder of experimental results in a natural frequency of $545 \mathrm{~Hz}$ cannot be calculated in theoretical calculations just like in Table 2. before improvement. However, compared with the experimental results, error between theoretical and experimental models is small, which proves rationality of the theoretical model and improvement.

Analyzing theoretical and experimental natural frequencies before and after improvement, closeness of natural frequency of each order decreases after improvement, but closeness of first-order is good. Obviously, first-order natural frequency changes from $248 \mathrm{~Hz}$ to $463 \mathrm{~Hz}$ showing a good stiffness improvement. In addition, first-order natural frequency of mode shapes has also changed, which is a change from vibration of dynamometer in $\mathrm{Z}$ direction to rotation of dynamometer along $\mathrm{Z}$ direction. This is because if seriesparallel model has changed, not only $k_{2 z}$ but also $k_{2 y}, k_{2 x}$ change, resulting in mode shapes change.

\section{CONCLUSIONS}

Taking dynamic characteristic of thrust vector control engine as research object, a novel dynamic design method is proposed to improve first-order natural frequency based on stiffness improvement. The conclusions are as follows: Based on characteristic in six degree-of-freedom of VFTD, kinetic model of dynamometer, rigid support, and other connecting devices are constructed with the Lagrange dynamic method to obtain theoretical natural frequency and mode shapes. Experimental natural frequency is obtained adopting the hammering method. Compared with theoretical results, maximum natural frequency error of each order is $5.96 \%$, which proves rationality of the Lagrange method. Law of first-order natural frequency over stiffness is analyzed with the Lagrange dynamic method, showing that first-order natural frequency converges to a constant value with increase of stiffness. By using the method of TECF, stiffness intervals of three-direction stiffness to satisfying requirement of natural frequency are obtained. New support devices are designed according to stiffness interval, which improves stiffness of TECF. Improved experimental natural frequency is obtained with improved structures, showing that first-order natural frequency has changed from $248 \mathrm{~Hz}$ to $463 \mathrm{~Hz}$, which verifies rationality of the dynamic design method. This dynamic method can be used to improve the first-order natural frequency in the test system, especially for large-mass system of low first-order natural frequency.

In a further stage of the research, the developed method for first-order natural frequency improvement with transfer function is expected to be complemented.

\section{ACKNOWLEDGMENT}

This project is supported by National Natural Science Foundation of China (Grant No. 51475078 and Grant No. 51675084), Aeronautical Science Foundation of China (Grant No. 20160163001) and Fundamental Research Funds for Chinese Central Universities (Grant No. DUT17GF211).

\section{REFERENCES}

[1] Liu, J., Meng, X.H., Zhang, D., Jiang, C., Han, X. (2017). An efficient method to reduce ill-posedness for structural dynamic load identification. Mechanical Systems and Signal Processing, 95, 273-285.

[2] Hessling, J.P. (2011). Propagation of dynamic measurement uncertainty. Measurement Science and Technology, 22 (10).

[3] Du, C., Zhang, J., Lu, D., Zhang, H., Zhao, W. (2016). A parametric modeling method for the pose-dependent dynamics of bi-rotary milling head. Proceedings of the Institution of Mechanical Engineers, Part B: Journal of Engineering Manufacture, 232, 797-811.

[4] Albrecht, A., Park, S.S., Altintas, Y., Pritschow, G. (2005). High frequency bandwidth cutting force measurement in milling using capacitance displacement sensors. International Journal of Machine Tools and Manufacture, 45, 993-1008.

[5] Li. Y.J., Wang, G.C., Zhang, J., Jia, Z.Y. (2012). Dynamic characteristics of piezoelectric sixdimensional heavy force/moment sensor for large-load robotic manipulator. Measurement, 45, 1114-1125.

[6] Qin, L., Jiang, C. (2011). Design and calibration of a novel piezoelectric six-axis force/torque sensor. In Seventh International Symposium on Precision Engineering Measurements and Instrumentation, SPIE 8321.

[7] Totis, G., Sortino, M. (2011). Development of a modular dynamometer for triaxial cutting force measurement in turning. International Journal of Machine Tools and Manufacture, 51, 34-42.

[8] Liu, X., Han, C., Wang, Y., Yang, T., Du, J., Zhu, M. (2016). Design of natural frequency adjustable electromagnetic actuator and active vibration control test. Journal of Low Frequency Noise, Vibration and Active Control, 35, 187-206.

[9] Scippa, A., Sallese, L., Grossi, N., Campatelli, G. (2015). Improved dynamic compensation for accurate cutting force measurements in milling applications. Mechanical Systems and Signal Processing, 54-55, 314-324.

[10] Ma, J., Song, A., Pan, D. (2013). Dynamic compensation for two-axis robot wrist force sensors. Journal of Sensors, 2013, art. ID 357396.

[11] Ren, Z.J., Sun, B.Y, Zhang, J., Qian, M. (2008). The dynamic model and acceleration compensation for the thrust measurement system of attitude/orbit rocket. In International Workshop on Modelling, Simulation and Optimization. IEEE, 30-33.

[12] Roj, J. (2013). Neural network based real-time correction of transducer dynamic errors. Measurement Science Review, 13 (6), 286-291.

[13] Law, M., Altintas, Y., Phani, A.S. (2013). Positiondependent multibody dynamic modeling of machine tools based on improved reduced order models. Journal of Manufacturing Science and Engineering, 135, 1-11. 
[14] Li, X., Wang, X., Wang, J. (2016). A kind of Lagrange dynamic simplified modeling method for multi-DOF robot. Journal of Intelligent \& Fuzzy Systems, 31, 2393 2401.

[15] Liu, M., Zhai, F., Chen, G., Li, Y., Guo, Z. (2016). Theoretical and experimental research on dynamics of the inner displaced indexing cam mechanism. Mechanism \& Machine Theory, 105, 620-632.

[16] Sang, W.L., Mayor, R., Ni, J. (2006). Dynamic analysis of a mesoscale machine tool. Journal of Manufacturing Science and Engineering, 128, 194-203.

[17] Schmitz, T.L., Donalson, R.R. (2000). Predicting highspeed machining dynamics by substructure analysis cirp. CIRP Annals - Manufacturing Technology, 49, 303-308.
[18] Wei, X.T. (2015). Vibration analysis and optimum design of electric vehicle powerplant mount system, Chongqing University, China, 1-6. (Master thesis in Chinese)

[19] Jia. X.H., Tian, Y.L., Zhang, D.W. (2010). Dynamic analysis of 3-prr compliant parallel mechanism. Transactions of The Chinese Society of Agricultural Machinery, 41, 199-203.

[20] Lu, H. (2007). Dynamic modeling and analysis of ultraprecision machine tool, Harbin Institute of Technology, China, 1-5. (Master thesis in Chinese)

[21] Liu, H.T., Zhao, W.H. (2010). Dynamic characteristic analysis for machine tools based on concept of generalized manufacturing space. Journal of Mechanical Engineering, 46, 54-60.

Received February 20, 2018 Accepted September 10, 2018 